\title{
Music, heart rate variability, and symptom clusters: a comparative study
}

\author{
Shu-Chuan Chen ${ }^{1,2} \cdot$ Ming-Lee Yeh ${ }^{3} \cdot$ Hsiu-Ju Chang ${ }^{4} \cdot$ Mei-Feng Lin $^{5}$ \\ Received: 22 January 2019 / Accepted: 16 April 2019 \\ (C) Springer-Verlag GmbH Germany, part of Springer Nature 2019
}

\begin{abstract}
Purpose This study aimed to explore the possible range of change of a single-session music intervention (SMI) on symptom clusters and neurological reactivity for women with breast cancer undergoing chemotherapy.

Methods A parallel and randomized, controlled study with repeated measures design was used. A total of 100 women with breast cancer were randomly assigned to the SMI or a control group. The outcome measurements of symptom cluster were collected using the Multidimensional Fatigue Symptom Inventory, Pittsburgh Sleep Quality Index, the Hospital Anxiety and Depression Scale, and the neurological reactivity with heart rate variability at four time points: before commencement of the intervention (T0), immediately afterward (T1), 1 week later (T2), and 3 weeks after the intervention (T3).

Results Of the 50 women in each group, 46 in the SMI and 48 in the control group completed the post-test at T3. Multivariate analysis of variance indicated that the SMI group had a medium effect in change of symptom clusters compared to the control group at T2. Moreover, after adjusting for baseline between normal and higher levels of sympathetic tone activity, significant differences existed in fatigue and depression at T2 and sleep disturbance at T3.

Conclusions A single-session music intervention can be effectively used to reduce symptom clusters for women with breast cancer. Targeting those who have a higher level of sympathetic tone activity is recommended.
\end{abstract}

Keywords Breast cancer · Fatigue - Sleep · Anxiety · Depression · Sympathetic tone activity

\section{Background}

Breast cancer $(\mathrm{BC})$ is the most common cancer in women in developed countries [1]. Women with BC obtained survival benefits from adjuvant chemotherapy that could significantly reduce the risk of mortality compared to women who do not receive chemotherapy [2]. However, chemotherapy can have adverse effects such as fatigue, sleep disturbance, and psychological distress that may co-occur $[3,4]$. A symptom cluster is

Mei-Feng Lin

L30mf@mail.ncku.edu.tw

Shu-Chuan Chen

shu-chuan.chen@griffithuni.edu.au

Ming-Lee Yeh

hsiuju@tmu.edu.tw

Hsiu-Ju Chang

hsiuju@ tmu.edu.tw

1 School of Nursing and Midwifery, Griffith University, Nathan, Brisbane, Queensland 4111, Australia three or more symptoms that occur together and are related to each other [5]. A high prevalence of symptom cluster, caused by the complexity of cancer itself and its treatment, impairs not only quality of life but also mood regulation of patients [6]. Therefore, it is expected that treating a single symptom may concurrently improve other symptoms [7].

A cluster of "fatigue-pain-depression-sleep disturbance" or "depression-sleep disturbance-fatigue" has been identified as the most common symptoms suffered in patients with

2 Department of Nursing, National Tainan Junior College of Nursing, Tainan, Taiwan, Republic of China

3 Department of Thanatology and Health Counseling, National Taipei University of Nursing and Health Science, No. 365, Ming-te Road, Peitou District, Taipei 11219, Taiwan, Republic of China

4 Department of Nursing, College of Nursing, Taipei Medical University, No.250, Wu-Hsing Street, Taipei 110, Taiwan, Republic of China

5 Department of Nursing, College of Medicine, National Cheng Kung University, No. 1, Tai-Hsueh Road, Tainan 701, Taiwan, Republic of China 
cancer by the review of Miaskowski et al. [8] and Sandadi et al. [9]. Sullivan et al. $[10,11]$ have reported that only five (i.e., psychological, hormonal) among eight symptom clusters were relatively stable in two dimensions (i.e., occurrence and severity) for women with $\mathrm{BC}$ undergoing chemotherapy over time. However, psychological clusters were ranked in the top three symptoms either in occurrence or severity ratings. Mechanisms that underlie symptom clusters is essential to developing targeted interventions were recommended by Miaskowski et al. [8]. Thus, we intended to better understand whether a "driving" symptom exists that triggers other symptoms in the cluster.

Results of a Cochrane review [12] reported that music interventions have beneficial effects on anxiety, depression, pain, fatigue, physiological responses, and quality of life. A study of single-session music therapy has revealed a greater positive effect on post-chemotherapy anxiety than that of the control group [13]. Music used as a protective strategy for improving sleep disturbance [14], reducing fatigue [15], anxiety [16], and depression [17] in patients with cancer have been validated separately. However, the studies examined the effect of music intervention on a single symptom rather than on symptom clusters; whether a single-session music intervention (SMI) could release chemotherapy-related symptom clusters simultaneously for cancer patients still lacks deep exploration. Given that 10 to $20 \%$ of women with BC reported the occurrence of anxiety and depression, Sullivan et al. [11] suggested these symptom clusters warrant careful management.

Musical reactions are recognized by the amygdala and hippocampus structures which are next to a hypothalamuspituitary axis centrally placed in the limbic system [18]. Soothing music's significant effects on the autonomous nervous system with the rhythm ranging from 60 to 80 beats per minute and the tempo is slow, to achieve a relaxing effect [19] and enhance mood, contributed to amygdala mediation [18]. As music rhythm and melody act on the peripheral and hippocampus system, relaxing and comforting music can be used to improve sleep quality, fatigue, and anxiety.

Heart rate variability (HRV), a physiological phenomenon of variation in the intervals between successive heartbeats [20], represents the complex interaction of neurological reactivity and reflects the sympathetic-parasympathetic modulation on the sinus node automaticity [21]. Evidence has shown the association of higher HRV parameters and prolonged survival, as well as higher levels of neuroendocrine markers with the symptom cluster of fatigue-depression-pain in cancer patients $[22,23]$. Meta-analysis results have shown that assessment of HRV could help in attenuation of adverse effects for women with BC such as fatigue, depression, and stress [24]. Another study demonstrated that overall cancer survivals were significantly longer in the higher HRV group than in the lower HRV group [25]. While many studies have been conducted on the relationship between HRV and survival rate in cancer patients [24, 25], few empirical studies have investigated whether HRV would affect the efficacy of music therapy for BC patients.

This current study aimed to fill the knowledge gap by exploring the possible range of change a SMI had on symptom clusters and neurological reactivity for women with BC undergoing chemotherapy. Our primary hypothesis was that the women who completed a SMI would exhibit a significantly decreased level of symptom clusters compared to the control group. The secondary hypothesis was that, after SMI, women with a higher level of sympathetic tone activity (HSTA) would present significantly improved symptom clusters compared to women with a normal level (NSTA).

\section{Methods}

\section{Study design}

A parallel, randomized, controlled study with repeated measures was used to examine the effects of a SMI on symptom clusters (i.e., fatigue-sleep disturbance-anxiety-depression) in women with $\mathrm{BC}$ during chemotherapy.

\section{Settings and participants}

This study was conducted in an outpatient clinic room for chemotherapy in a medical center with 1320 beds. Potential participants were identified by a $\mathrm{BC}$ case manager and formally screened against the described criteria by a trained research assistant (RA). Women with newly diagnosed stage 0-II BC who were scheduled to receive the first cyclic chemotherapy with six 3-week rounds were approached. Women were excluded if they have a diagnosis of neurological disorders, sleep apnea, and arrhythmia.

\section{Sample size}

The sample size was calculated based on a prior study that looked at the effect of music intervention on depression [17]. A sample size of 76-98 would achieve $80-90 \%$ power with 0.05 alpha to detect a difference of 0.7 in the hospital anxiety and depression scale score for a study design with repeated measures using PASS version 14 software [26]. To allow for a $20 \%$ attrition rate, this study sought to recruit a sample of 118 women with $\mathrm{BC}$. Of the 127 women with $\mathrm{BC}$ who met all eligibility criteria for the study, 27 women declined to participate due to lack of interest $(n=15)$, did not return consent forms and baseline questionnaires $(n=7)$, and did not provide reasons $(n=5)$. Of the remaining 100 women, 50 were allocated to either the SMI group or the control group (Fig. 1). 
Fig. 1 CONSORT flow diagram of the trial

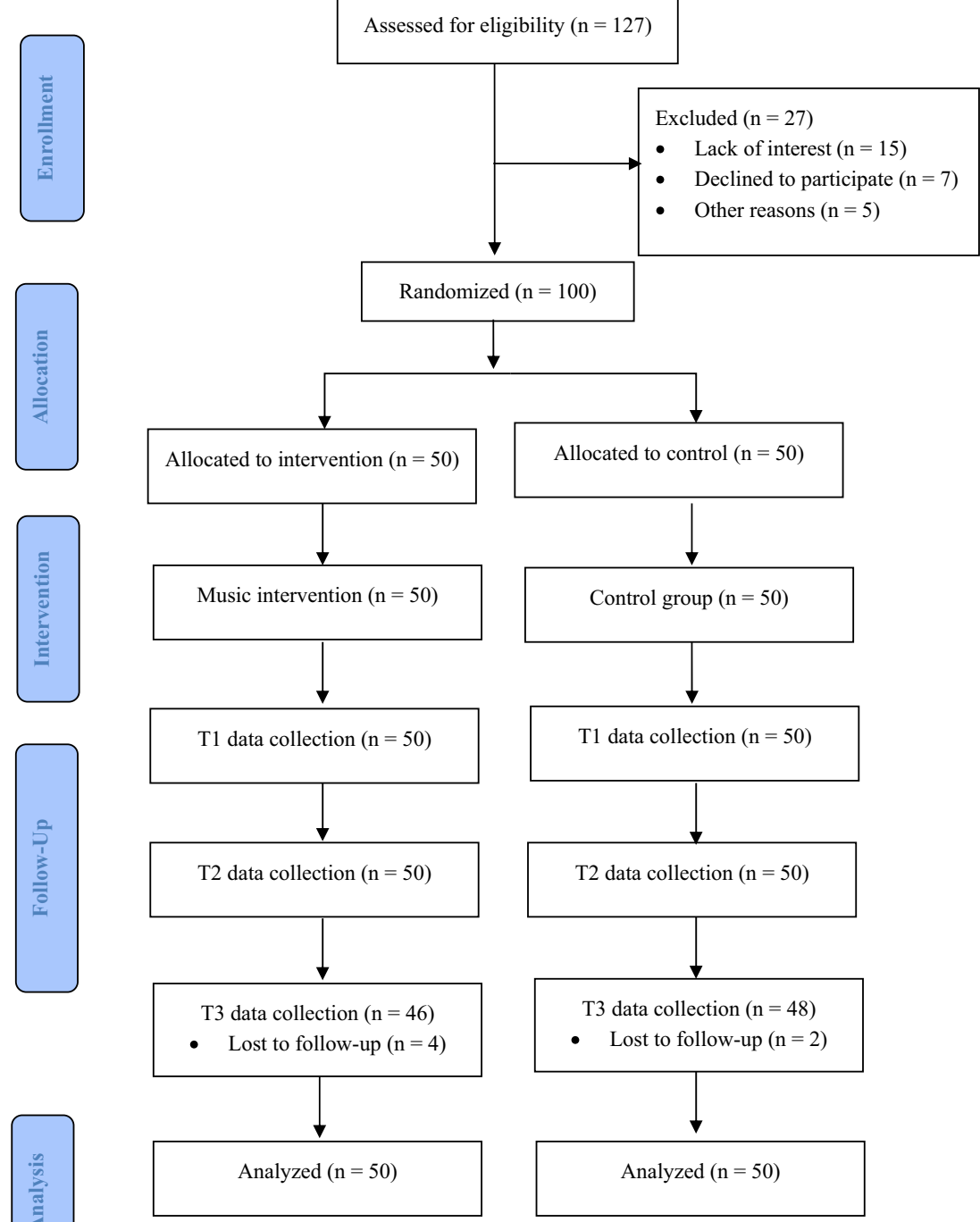

\section{Procedures}

Eligible participants were randomly allocated to the experimental and control group using a computer-generated sequence. Ethical approval was approved by the institutional review board of the participating hospital in Taiwan (B-ER-102-004). Questionnaire data were collected at the following four time points: before the intervention (T0), immediately after the intervention (T1), 1 week after the intervention (T2), and 3 weeks after the intervention (T3). The RAs administered questionnaires using face-to-face interviews at $\mathrm{T} 0$ and $\mathrm{T} 1$ and telephone interviews at T2 and T3. After completion of questionnaires at $\mathrm{T} 0$, spot electrodes were placed in the forearm and chest of each participant for electrocardiogram (ECG) to collect HRV data for $15 \mathrm{~min}$. The women were asked to refrain from speaking and moving and to rest comfortably in their chair for five-minute resting ECG data were recorded.

\section{Intervention and control groups}

The components of music intervention including content, delivery, interventionist and treatment fidelity were followed the guidelines developed by Robb et al. [27]. While undergoing chemotherapy, the women in the SMI group received a 45-min SMI by CD player with a headphone, delivered by a trained music therapist in a private clinical setting. Content with seven to eight pieces of recorded music including light music, sounds of nature, folk songs, pop songs, and classical music was given. The first two or three tracks were chosen according to participants' music preference from a database to act as a prelude for preparing to embark on a musical journey. The music sets were built 
from the results of studies used to alleviate anxiety [13], depression [17], or improve sleep quality [28].

Women in the control group received routine nursing care but no music intervention during their chemotherapy.

\section{Outcome measures}

Demographics, disease-related characteristics, and HRV were collected from the clinical records before chemotherapy. The outcome of interest was changes in level of symptom clusters including fatigue, sleep disturbance, anxiety, and depression. Outcomes were assessed at the four time points described above. Data were collected between 2013 and 2015.

\section{Demographic data and disease-related characteristics}

Demographic data included age, marital status, and level of education. Disease-related characteristics were obtained from clinical records including stage of cancer, type of surgery, type of treatment regimen, frequency of chemotherapy, and taking of sedative medications.

\section{MFSI-SF}

Fatigue was assessed by the MFSI-SF, which consisted of 30 items with 5 subscales: general, physical, emotional, mental, and vigor [29]. Each subscale consists of 6 items, and each item is rated on a 5 -point Likert-type scale $(0=$ not at all; $4=$ extremely). The sum of four subscale scores (i.e., general, physical, emotional, and mental) minus the vigor subscale score yields the total score. Higher scores indicate more severe fatigue. Reliability for the five subscales was assessed in a cancer study [30] and in the present study (alpha) ranged from .78 to .90 .

\section{PSQI-T}

Sleep quality was measured with the 19-item Pittsburgh Sleep Quality Index-Taiwan Form (PSQI-T) translated by Wang and colleagues [28]. It is a self-report questionnaire assessing type and frequency of sleep disturbances experienced over 1 month. The PSQI-T includes seven components: subjective sleep quality, sleep latency, duration, habitual sleep efficiency, sleep disturbances, use of sleeping medication, and daytime dysfunction [31]. Scores higher than five indicate poor sleep quality [31]. Cronbach's alphas of internal consistency were $.79-.91$ in a sample of Taiwanese patients with cancer [32] and .66 in this study.

\section{HADS}

Psychological distress was assessed using the Hospital Anxiety and Depression Scale (HADS), a self-assessment scale developed as a reliable instrument for detecting states of depression and anxiety in people with medical illness [33]. The full 14-item rating scale with 2 subscales contained 7 items each for anxiety (HADS-A) and depression (HADSD), respectively. Participants were asked to rate their depressive or distressed status on a 4-point Likert-type scale ranging from 0 to 3 . The higher the score, the more severe the anxiety or depression is. Scores range from 0 to 21 for each subscale and are divided into 4 levels [34]. The HADS has been used extensively in Taiwan's BC populations [17]. In this current study, Cronbach's alpha of internal consistency reliability was .79 in HADS-A and .65 in HADS-D.

\section{Heart rate variability}

HRV serves as an indicator of activity response and relaxation of the sympathetic nervous system. It was collected in this study using the Wireless Physiological Feedback System (Model NeXus-4) manufactured by Mind Media in the Netherlands. This device sets 400 signal acquisitions per minute and regularly corrects the device to maintain good reliability. Signal acquisition is managed, corrected, and heart rate frequency calculated by BioTrace+ software (Mind Media). Autonomic neurophysiological status was reflected by the amplitude of high-frequency (HF) and low-frequency (LF) waveforms that were analyzed with the LF/HF ratio [35].

\section{Statistical methods}

Statistical analysis SPSS version 23 was used, and an intention-to-treat analysis was used in line with the CONSORT statement. Descriptive variables were analyzed with frequencies, percentages, means, and standard deviations. The chi-square test and independent $t$ tests were used to test for group differences in terms of demographics and disease-related variables at baseline. A multivariate analysis of variance (MANOVA) was used to compare changes of outcome measures at each time point, with corresponding baseline scores and the taking of sedative medications entered as covariates. It was also used to explore the impacts of music intervention on symptom clusters between subgroups of NSTA and HSTA with adjusting baseline scores as covariates. Statistically significant levels were considered with two-tailed $p$ values $<.05$.

\section{Results}

A total of 100 women completed the questionnaires at baseline, with 6 women (6\%) lost to follow-up at T3 (Fig. 1). A summary of the demographic and disease- 
Table 1 Demographic characteristics of the two group patients $(n=100)$

\begin{tabular}{|c|c|c|c|c|c|c|}
\hline \multirow{2}{*}{$\frac{\text { Variable }}{\text { Age(years) (mean (SD)) }}$} & \multirow{2}{*}{$\begin{array}{l}\text { Experimental group } \\
51.16(9.21)\end{array}$} & \multicolumn{2}{|c|}{ Control group } & \multirow{2}{*}{$\frac{t / \chi^{2}}{-0.84 *}$} & \multicolumn{2}{|l|}{$p$} \\
\hline & & \multicolumn{2}{|c|}{$49.56(9.78)$} & & \multicolumn{2}{|l|}{.40} \\
\hline \multicolumn{7}{|l|}{ Education level } \\
\hline Primary school & 12 & 24 & 6 & 12 & \multirow[t]{3}{*}{6.43} & \multirow[t]{3}{*}{.09} \\
\hline Junior high school & 24 & 48 & 23 & 48 & & \\
\hline College and above & 14 & 28 & 20 & 40 & & \\
\hline \multicolumn{7}{|l|}{ Marital status $(n, \%)$} \\
\hline Married & 35 & 70 & 42 & 84 & & \\
\hline Single/widow/divorced & 15 & 30 & 8 & 16 & 2.78 & .15 \\
\hline \multicolumn{7}{|l|}{ Cancer stage $(n, \%)$} \\
\hline $0, \mathrm{I}$ & 12 & 24 & 14 & 28 & \multirow[t]{3}{*}{0.25} & \multirow[t]{3}{*}{.88} \\
\hline II & 17 & 34 & 17 & 34 & & \\
\hline III, IV & 21 & 42 & 19 & 38 & & \\
\hline \multicolumn{7}{|l|}{ Surgery $(n, \%)$} \\
\hline None & 6 & 12 & 7 & 14 & & \\
\hline $\mathrm{PM}$ & 15 & 30 & 9 & 18 & & \\
\hline $\mathrm{TM} / \mathrm{MRM}$ & 29 & 58 & 34 & 68 & 1.97 & .37 \\
\hline \multicolumn{7}{|l|}{ Treatment regimen $(n, \%)$} \\
\hline TEC & 14 & 28 & 15 & 30 & & \\
\hline FEC & 16 & 32 & 16 & 32 & & \\
\hline $\mathrm{TCH} / \mathrm{CE}$ & 20 & 40 & 19 & 38 & 0.06 & .97 \\
\hline \multicolumn{7}{|c|}{ Sedative medication use $(n, \%)$} \\
\hline Yes & 0 & 0 & 17 & 34 & \multirow[t]{2}{*}{20.48} & \multirow[t]{2}{*}{$<.001 * *$} \\
\hline No & 50 & 100 & 33 & 66 & & \\
\hline \multicolumn{7}{|c|}{ Sympathetic tone activity $(n, \%)$} \\
\hline NSTA & 34 & 68 & 32 & 64 & \multirow[t]{2}{*}{0.18} & \multirow[t]{2}{*}{.67} \\
\hline HSTA & 16 & 32 & 18 & 36 & & \\
\hline \multicolumn{7}{|l|}{ Rounds of CT $(n, \%)$} \\
\hline $1 \mathrm{st}$ & 34 & 68 & 35 & 70 & \multirow{3}{*}{3.20} & \multirow{3}{*}{.36} \\
\hline 2nd & 13 & 26 & 8 & 16 & & \\
\hline $3 \mathrm{rd} / 4 \mathrm{th} / 5 \mathrm{th} / 6 \mathrm{th}$ & 3 & 6 & 7 & 14 & & \\
\hline
\end{tabular}

$t t$ test, $\chi^{2}$ chi-square, $P M$ partial mastectomy, MRM modified radical mastectomy, $T M$ total mastectomy, TEC docetaxel, epirubicin, cyclophosphamide, FEC 5-FU, epirubicin, cyclophosphamide, $E C$ epirubicin, cyclophosphamide, TCH docetaxel, carboplatin, trastuzumab, NSTA normal level of sympathetic tone activity, HSTA higher level of sympathetic tone activity, $C T$ chemotherapy

$* * p<.05$

related characteristics of participants is presented in Table 1. The majority were married, education level was at junior high school or above, and diagnosed of BC stage I or II. Approximately one-third of the women in both groups showed higher levels of sympathetic tone activity. No significant differences existed in both groups at baseline (all $p s>.05$ ) except for the taking of sedative medications by 17 (34\%) women in the control group $(p<.001)$. Those medications included antihistamines, major and minor tranquilizers, and sedative hypnotics. Therefore, taking sedative medication as a covariate was taken into account in subsequent analysis. Differences between the two groups were not significant in each variable at T0 (Table 2).

\section{The range of change from a single-session of music intervention}

The primary hypothesis was partially supported; the women who completed SMI exhibited significantly decreased symptom clusters at T2 compared to the control group, with adjusting baseline scores and the taking of sedative medications as covariates. Preliminary assumption testing was conducted to check for normality, linearity, univariate and multivariate outliers, homogeneity of variable covariate matrices, and multicollinearity, with no serious violation noted. No significance on symptom clusters (fatigue-sleep disturbanceanxiety-depression) at T1 and T3 was examined by a oneway MANOVA (Table 3). The results revealed a significant 
Table 2 Changes of outcome measures at each time point compared to baseline between two groups $(n=100)$

\begin{tabular}{|c|c|c|c|c|c|c|c|}
\hline \multirow[t]{2}{*}{ Variable } & \multirow[t]{2}{*}{ Time } & \multicolumn{2}{|l|}{ SMI } & \multicolumn{2}{|c|}{ Control } & \multirow[t]{2}{*}{$t$} & \multirow[t]{2}{*}{$p$} \\
\hline & & Mean & SD & Mean & SD & & \\
\hline \multirow[t]{4}{*}{ MFSI-SF } & T0 & 11.64 & 17.25 & 5.74 & 15.57 & \multirow[t]{4}{*}{-1.80} & \multirow[t]{4}{*}{.08} \\
\hline & $\mathrm{T} 1$ & 9.26 & 17.09 & 7.00 & 14.22 & & \\
\hline & $\mathrm{T} 2$ & 19.42 & 18.34 & 12.35 & 17.72 & & \\
\hline & $\mathrm{T} 3$ & 11.80 & 17.42 & 8.39 & 17.42 & & \\
\hline \multirow[t]{4}{*}{ PSQI-T } & T0 & 8.67 & 2.62 & 8.70 & 4.39 & \multirow[t]{4}{*}{0.04} & \multirow[t]{4}{*}{.97} \\
\hline & $\mathrm{T} 1$ & 8.38 & 3.69 & 8.78 & 4.93 & & \\
\hline & $\mathrm{T} 2$ & 8.96 & 4.16 & 9.50 & 5.13 & & \\
\hline & $\mathrm{T} 3$ & 7.62 & 3.42 & 8.77 & 4.99 & & \\
\hline \multirow[t]{4}{*}{ HADS-A } & T0 & 6.46 & 4.08 & 5.60 & 3.65 & \multirow[t]{4}{*}{-1.11} & \multirow[t]{4}{*}{.27} \\
\hline & $\mathrm{T} 1$ & 5.78 & 4.20 & 5.06 & 3.78 & & \\
\hline & $\mathrm{T} 2$ & 6.62 & 3.82 & 5.60 & 3.86 & & \\
\hline & $\mathrm{T} 3$ & 5.18 & 4.17 & 5.02 & 3.55 & & \\
\hline \multirow[t]{4}{*}{ HADS-D } & T0 & 8.94 & 3.28 & 8.68 & 2.31 & \multirow[t]{4}{*}{-0.46} & \multirow[t]{4}{*}{.65} \\
\hline & $\mathrm{T} 1$ & 8.60 & 2.97 & 8.30 & 2.87 & & \\
\hline & $\mathrm{T} 2$ & 10.12 & 2.91 & 8.83 & 3.09 & & \\
\hline & $\mathrm{T} 3$ & 9.22 & 2.78 & 8.82 & 3.19 & & \\
\hline
\end{tabular}

$p<.05$

$M F S I-S F$ the Multidimensional Fatigue Symptom Inventory-Short Form, PSQI-T Pittsburgh Sleep Quality Index-Taiwan Form, HADS-A the Hospital Anxiety and Depression Scale-Anxiety, HADS-D the Hospital Anxiety and Depression Scale-Depression, $T O$ before the commencement of the intervention, $T 1$ immediately after intervention, $T 2$ 1 week after intervention, T3 3 weeks after intervention

difference between SMI and the control group on the symptom clusters at $\mathrm{T} 2$ with a medium effect size $\left(F_{4,91}=3.07\right.$, $p=.02$, Wilks $\lambda=0.88, \eta p^{2}=.12$ ). When results for the dependent variables were considered separately, sleep disturbance and anxiety reached statistical significance at both $\mathrm{T} 1$ and $\mathrm{T} 3$ with medium effect sizes.

\section{The HSTA subsample that benefitted most from a single-session of music intervention}

The secondary hypothesis was also partially supported. In Table 4, a MANOVA was conducted to compare the effects of SMI between the NSTA and HSTA subgroups at each time point. Preliminary checks were conducted to ensure that there was no violation of the assumptions. The results revealed that there was no statistical significance between the HSTA and NSTA subgroups on the combined symptom clusters at each of the four time points, after adjusting for baseline score. However, when the results for the dependent variables were considered separately, significant differences existed between the HSTA and NSTA subgroups with medium effect sizes in fatigue $\left(F_{1,44}=5.42, p=.02 ; \eta p^{2}=.11\right)$ and depression $\left(F_{1}\right.$, $\left.44=4.99, p=.03 ; \eta p^{2}=.1\right)$ at T2, as well as sleep disturbance
$\left(F_{1,44}=6.5, p=.01 ; \eta p^{2}=.13\right)$ at T3. No significant differences were found between HSTA and NSTA in the control group (all $p \mathrm{~s}>.05$ ).

\section{Discussion}

This present study examined the possible range of change in reducing symptom clusters caused by a SMI among women with BC. It was demonstrated that a SMI is an effective strategy in reducing symptom clusters (fatigue-sleep disturbanceanxiety-depression) for women undergoing chemotherapy, with medium effect size at T2 and sleep disturbance and anxiety at $\mathrm{T} 1$ and $\mathrm{T} 3$, respectively. Although women receiving a 45-min music intervention did not show significant difference on symptom clusters between the NSTA and HSTA subgroups at four time points, women with a higher level of sympathetic tone activity reduced fatigue and depression at $\mathrm{T} 2$ and improved sleep disturbance at T3, separately.

\section{Effect of SMI on symptom clusters}

Women with $\mathrm{BC}$ receiving SMI achieved significantly reduced symptom clusters after 1 week of chemotherapy and improved the individual symptoms of anxiety and sleep disturbance immediately and 3 weeks after chemotherapy but had no significant change in fatigue and depression.

The most important clinically relevant findings of this study were that SMI has significantly improved the symptom clusters (four symptoms) after 1 week of chemotherapy. This might relate to the trajectories of symptom clusters in women with BC. Previous studies have shown that Taiwanese women with $\mathrm{BC}$ undergoing chemotherapy experienced the most severe symptoms in the first week after chemotherapy [36, 37]. This present study demonstrated that SMI could help to reduce severity of symptom clusters, particularly 1 week after chemotherapy, the most uncomfortable period. These findings are consistent with previous studies that stated music-based intervention enhanced the comfort of cancer patients [16, 38]. Consequently, this present study supports that women receiving SMI have greater reduction of symptom clusters compared with the control group. Studies have revealed that some symptoms might overlap and share a common etiology, such as fatigue and sleep disturbances [39, 40]. Hence, once a symptom was improved, other symptoms might be improved simultaneously as well. Therefore, to integrate and improve quality of care using music intervention as a treatment strategy for women with BC, clinical intervention studies need to focus on symptom clusters rather than a single symptom. Additionally, these results concur with previous studies that showed musicbased interventions have an immediate effect in anxiety 
Table 3 MANOVA with covariate of outcome measures between SMI and the control groups at each time point $(N=$ 100)

\begin{tabular}{|c|c|c|c|c|c|c|c|c|c|c|c|}
\hline \multirow[b]{2}{*}{ Time } & \multirow[b]{2}{*}{ Variable } & \multicolumn{5}{|c|}{ Combined variables } & \multicolumn{5}{|c|}{ Separate variable } \\
\hline & & Wilks' $\lambda$ & $F$ & df & $p$ & $\eta p^{2}$ & MS & $F$ & df & $p$ & $\eta p^{2}$ \\
\hline \multirow[t]{4}{*}{ T0 } & MSFI & .79 & 6.25 & 4,93 & .00 & .21 & 2516.03 & 10.55 & 1,96 & .00 & .10 \\
\hline & PSQI & & & & & & 119.47 & 17.05 & 1,96 & .00 & .15 \\
\hline & HAD-A & & & & & & 73.84 & 5.25 & 1,96 & .02 & .05 \\
\hline & HAD-D & & & & & & 12.24 & 1.55 & 1,96 & .22 & .02 \\
\hline \multirow[t]{4}{*}{$\mathrm{T} 1$} & MSFI & .91 & 2.20 & 4,94 & .07 & .09 & 896.27 & 3.90 & 1,97 & .05 & .04 \\
\hline & PSQI & & & & & & 83.27 & 6.32 & 1,97 & $.01 *$ & .06 \\
\hline & HAD-A & & & & & & 78.89 & 5.44 & 1,97 & $.02 *$ & .05 \\
\hline & HAD-D & & & & & & 9.49 & 1.12 & 1,97 & .29 & .01 \\
\hline \multirow[t]{4}{*}{$\mathrm{T} 2$} & MSFI & .88 & 3.07 & 4,91 & $.02 *$ & .12 & 2217.80 & 8.47 & 1,94 & $.00^{*}$ & .08 \\
\hline & PSQI & & & & & & 94.65 & 5.76 & 1,94 & $.02 *$ & .06 \\
\hline & HAD-A & & & & & & 149.45 & 10.50 & 1,94 & $.00 *$ & .10 \\
\hline & HAD-D & & & & & & 39.00 & 4.09 & 1,94 & $.05^{*}$ & .04 \\
\hline \multirow[t]{4}{*}{$\mathrm{T} 3$} & MSFI & .91 & 2.20 & 4,94 & .07 & .09 & 896.27 & 3.90 & 1,97 & .05 & .04 \\
\hline & PSQI & & & & & & 83.27 & 6.32 & 1,97 & $.01 *$ & .06 \\
\hline & HAD-A & & & & & & 78.89 & 5.44 & 1,97 & $.02 *$ & .05 \\
\hline & HAD-D & & & & & & 9.49 & 1.12 & 1,97 & .29 & .01 \\
\hline
\end{tabular}

MFSI-SF the Multidimensional Fatigue Symptom Inventory-Short Form, PSQI-T Pittsburgh Sleep Quality Index-Taiwan Form, HADS-A the Hospital Anxiety and Depression Scale-Anxiety, HADS-D the Hospital Anxiety and Depression Scale-Depression, $T 0$ before the commencement of the intervention, $T 1$ immediately after intervention, $T 21$ week after intervention, $T 33$ weeks after intervention, Wilks' $\lambda$ Wilks' lambda, $\eta p^{2}$ partial eta squared

*Adjusted baseline score $p<.05$ reduction [17, 41] and sleep disturbance [42]. Given that music intervention can help with immediate physical relaxation [17], anxiety and sleep disturbance were physical symptoms that responded more quickly to the music intervention than did psychological distress. This might be the reason that music intervention could improve anxiety and sleep disturbance immediately.

These findings raised intriguing questions regarding the nature of chemotherapy-related symptoms and the extent of a SMI for women with BC. Previous studies focused merely on individual symptoms to seek out a treatment strategy; however, this could have overemphasized or amplified the effect of a single symptom for women with cancer. This study's combination of findings provides some support for the premise that adopting the symptom cluster approach to manage chemotherapyrelated symptoms is a better strategy in clinical practice. In this study, the simultaneous existence of four symptom clusters was examined statistically by MANOVA, which can reduce the repeatability of independent interphase measurement. This result is based on a magnitude of effect size of a SMI to establish an intervention strategy of symptom clusters in music intervention, which is considered from the holistic care for women with BC. However, further studies need to take these variables into account.

\section{The HSTA subsample that benefitted most from music intervention}

In this study, we found that, although there were no significant differences on combined symptom clusters between NSTA and HSTA subgroups after receiving music intervention, women with HSTA significantly decreased their level of fatigue and depression at T2 and sleep disturbance at T3 compared to women with NSTA. The results echoed previous studies that revealed an association between HRV and fatigue among cancer patients [43, 44]. In the study of Lin et al. [13], patients with higher anxiety receiving music therapy had significantly decreased anxiety post-chemotherapy than did those with normal anxiety. Our study continuously explored the neurological reactivity of patients and demonstrated that women with a higher level of sympathetic tone activity had a better response to SMI; this group may be the subsample most benefitted by music intervention. This result is supported by Thayer and Sternberg [45], who articulated that higher parasympathetic activity (PA) facilitates energy conservation; however, prolonged heightened sympathetic activity (SA) creates disproportionate energy demands on the body. Accordingly, sympathetic overactivity and parasympathetic underactivity may be important biomarkers of cancer-related fatigue and may also have an etiological role [43, 44]. 
Table 4 MANOVA with covariate of outcome measures between NSTA and HSTA subgroups at each time point $(n=$ 50)

\begin{tabular}{|c|c|c|c|c|c|c|c|c|c|c|c|}
\hline \multirow[t]{2}{*}{ Time } & \multirow[t]{2}{*}{ Variable } & \multicolumn{5}{|c|}{ Combined variables } & \multicolumn{5}{|c|}{ Separate variable } \\
\hline & & Wilks' $\lambda$ & $F$ & $\mathrm{df}$ & $p$ & $\eta p^{2}$ & MS & $F$ & $\mathrm{df}$ & $p$ & $\eta p^{2}$ \\
\hline \multirow[t]{4}{*}{ T0 } & MSFI & 0.88 & 1.55 & 4,45 & .20 & .12 & 740.52 & 2.57 & 1,48 & .12 & .05 \\
\hline & PSQI & & & & & & 16.60 & 2.55 & 1,48 & .12 & .05 \\
\hline & HAD-A & & & & & & 14.68 & 0.88 & 1,48 & .35 & .02 \\
\hline & HAD-D & & & & & & 36.62 & 3.59 & 1,48 & .06 & .07 \\
\hline \multirow[t]{4}{*}{$\mathrm{T} 1$} & MSFI & 0.95 & 0.54 & 4,41 & .71 & .05 & 7.66 & 0.04 & 1,44 & .85 & .00 \\
\hline & PSQI & & & & & & 15.92 & 1.59 & 1,44 & .21 & .03 \\
\hline & HAD-A & & & & & & 0.31 & 0.04 & 1,44 & .85 & .00 \\
\hline & HAD-D & & & & & & 0.32 & 0.05 & 1,44 & .82 & .00 \\
\hline \multirow[t]{4}{*}{$\mathrm{T} 2$} & MSFI & 0.84 & 2.00 & 4,41 & .11 & .16 & 1377.69 & 5.42 & 1,44 & $.02 *$ & .11 \\
\hline & PSQI & & & & & & 10.79 & 0.80 & 1,44 & .37 & .02 \\
\hline & HAD-A & & & & & & 20.55 & 2.04 & 1,44 & .16 & .04 \\
\hline & HAD-D & & & & & & 30.45 & 4.99 & 1,44 & $.03 *$ & .10 \\
\hline \multirow[t]{4}{*}{ T3 } & MSFI & 0.84 & 1.96 & 4,41 & .12 & .16 & 170.87 & 0.82 & 1,44 & .37 & .02 \\
\hline & PSQI & & & & & & 52.69 & 6.50 & 1,44 & $.01 *$ & .13 \\
\hline & HAD-A & & & & & & 23.34 & 2.17 & 1,44 & .15 & .05 \\
\hline & HAD-D & & & & & & 8.83 & 1.64 & 1,44 & .21 & .04 \\
\hline
\end{tabular}

MFSI-SF Multidimensional Fatigue Symptom Inventory — Short Form, PSQI-T Pittsburgh Sleep Quality IndexTaiwan Form, HADS-A Hospital Anxiety and Depression Scale-Anxiety, HADS-D Hospital Anxiety and Depression Scale-Depression, $T 0$ before commencement of the intervention, $T 1$ immediately after intervention, T2 1 week after intervention, T3 3 weeks after intervention, Wilks' $\lambda$ Wilks' lambda, $\eta p^{2}$ partial eta squared

*Adjusted baseline score $p<.05$
However, this detailed mechanism is still inconsistent in the present literature; these results therefore need to be interpreted with caution. This study was the first to explore the relationship of music, HRV, and fatigue, and further exploration is needed of the impact of multiple music interventions on these combined variables.

\section{Limitations and strengths}

This study had some limitations. First, our data demonstrated that there were significant differences in sleep disturbance, anxiety, and fatigue at baseline although we did randomly allocate participants and use statistical techniques to deal with the situation. The outcome, however, should be interpreted with caution. Second, although data on medication use were collected, information on whether a medication was taken as a treatment for insomnia or anxiety was not recorded. Moreover, we did not collect data concerning other types of medication taken such as antidepressants, Chinese medicines or other herbs for psychological distress purposes. Finally, the low Cronbach's alpha of the PSQI-T and HADS requires retesting in a further study.

Conversely, this study had some strengths. First, this study confirmed that symptom clusters can be managed simultaneously. A short-term music intervention could be included in treatment plans and incorporated as an essential component of cancer nursing care. Second, for women with a HSTA, music intervention could be used as an effective intervention to help them reduce fatigue during chemotherapy. Therefore, these results suggest that SMI could be developed as a standard operating procedure for oncological units. To develop a full picture of music intervention, additional studies are needed to determine how many doses of music intervention will help to improve chronic symptom clusters for patients with cancer.

\section{Conclusion}

A single 45-min session of music intervention can serve as an effective strategy to reduce symptom cluster in women with BC after 1 week of chemotherapy. It also can individually alleviate anxiety and sleep disturbance with a medium effect immediately and at 3 weeks after chemotherapy. Women with higher neurological reactivity had a better response to the SMI. A SMI potentially decreases the development of fatigue in women with $\mathrm{BC}$; targeting particularly those who have higher levels of sympathetic tone activity is recommended. To enhance our understanding of such clinical changes, future studies should include an examination of the mechanisms among music, HRV, and cancer-related symptom clusters. A 
further study with more focus on the impact of music intervention on patients with higher neurological reactivity that can propose an explanation for effective changes is therefore suggested.

Acknowledgements We acknowledge the assistance from participants and the breast cancer care team of NCKUH in Taiwan.

Statement of authorship All persons who meet authorship criteria are listed as authors. Authorship contributions: Conception and design of study (Chen and Lin), acquisition of data (Chen and Lin), analysis and interpretation of data (Chen and Lin), drafting the manuscript (Chen and Lin), revising the manuscript critically for important intellectual content (all authors), and approval of the version of the manuscript to be published (all authors).

Funding Funding support from the Minister of Science Technology, Taiwan, R.O.C. (MOST 102-2410-H-006 -013).

\section{Compliance with ethical standards}

Informed consent was obtained by principle investigator before the commencement of the study.

Conflict of interest The authors declare that there is no conflict of interest.

Ethical approval The study had been approved by the Institutional Review Board of National Cheng Kung University Hospital (B-ER102-004).

\section{References}

1. World Health Organization (2018) Breast cancer: prevention and control. http://www.who.int/cancer/detection/breastcancer/en/ index1.html. Accessed 05 June 2018

2. Early Breast Cancer Trialists' Collaborative Group (EBCTCG) (2018) Long-term outcomes for neoadjuvant versus adjuvant chemotherapy in early breast cancer: meta-analysis of individual patient data from ten randomised trials. Lancet Oncol 19(1):27-39. https://doi.org/10.1016/s1470-2045(17)30777-5

3. Ho S-Y, Rohan KJ, Parent J, Tager FA, McKinley PS (2015) A longitudinal study of depression, fatigue, and sleep disturbances as a symptom cluster in women with breast cancer. J Pain Symptom Manag 49(4):707-715. https://doi.org/10.1016/j. jpainsymman.2014.09.009

4. Nguyen J, Cramarossa G, Bruner D, Chen E, Khan L, Leung A, Lutz S, Chow E (2011) A literature review of symptom clusters in patients with breast cancer. Expert Rev Pharmacoecon Outcomes Res 11(5):533-539. https://doi.org/10.1586/erp.11.55

5. Dodd MJ, Miaskowski C, Paul SM (2001) Symptom clusters and their effect on the functional status of patients with cancer. Oncol Nurs Forum 28(3):465-470

6. Dantzer R, Meagher MW, Cleeland CS (2012) Translational approaches to treatment-induced symptoms in cancer patients. Nat Rev Clin Oncol 9(7):414-426. https://doi.org/10.1038/nrclinonc. 2012.88

7. Donovan KA, Jacobsen PB (2007) Fatigue, depression, and insomnia: evidence for a symptom cluster in cancer. Semin Oncol Nurs 23(2):127-135
8. Miaskowski C, Barsevick A, Berger A, Casagrande R, Grady PA, Jacobsen P, Kutner J, Patrick D, Zimmerman L, Xiao CH, Matocha M, Marden S (2017) Advancing symptom science through symptom cluster research: expert panel proceedings and recommendations. J Natl Cancer Inst 109(4):djw253. https://doi.org/10.1093/ jnci/djw253

9. Sandadi S, Frasure HE, Broderick MJ, Waggoner SE, Miller JA, von Gruenigen VE (2011) The effect of sleep disturbance on quality of life in women with ovarian cancer. Gynecol Oncol 123(2):351355. https://doi.org/10.1016/j.ygyno.2011.07.028

10. Ward Sullivan CRNP, Leutwyler HRNP, Dunn LBMD, Cooper BAP, Paul SMP, Conley YPP, Levine JDMDP, Miaskowski CARNP (2017) Differences in symptom clusters identified using symptom occurrence rates versus severity ratings in patients with breast cancer undergoing chemotherapy. Eur J Oncol Nurs 28:122132. https://doi.org/10.1016/j.ejon.2017.04.001

11. Sullivan CW, Leutwyler H, Dunn LB, Cooper BA, Paul SM, Levine JD, Hammer M, Conley YP, Miaskowski CA (2018) Stability of symptom clusters in patients with breast cancer receiving chemotherapy. J Pain Symptom Manag 55(1):39-55. https:// doi.org/10.1016/j.jpainsymman.2017.08.008

12. Bradt J, Dileo C, Magill L, Teague A (2016) Music interventions for improving psychological and physical outcomes in cancer patients. Cochrane Database Syst Rev (8):Cd006911. doi:https://doi. org/10.1002/14651858.CD006911.pub3

13. Lin M-F, Hsieh Y-J, Hsu Y-Y, Fetzer S, Hsu M-C (2011) A randomised controlled trial of the effect of music therapy and verbal relaxation on chemotherapy-induced anxiety. J Clin Nurs 20(7/8): 988-999. https://doi.org/10.1111/j.1365-2702.2010.03525.x

14. Feng F, Zhang Y, Hou J, Cai J, Jiang Q, Li X, Zhao Q, Li BA (2017) Can music improve sleep quality in adults with primary insomnia? A systematic review and network meta-analysis. Int J Nurs Stud 77: 189-196. https://doi.org/10.1016/j.ijnurstu.2017.10.011

15. Tsai HF, Chen YR, Chung MH, Liao YM, Chi MJ, Chang CC, Chou KR (2014) Effectiveness of music intervention in ameliorating cancer patients' anxiety, depression, pain, and fatigue: a metaanalysis. Cancer Nurs 37(6):E35-E50. https://doi.org/10.1097/ NCC. 0000000000000116

16. Bilgic S, Acaroglu R (2017) Effects of listening to music on the comfort of chemotherapy patients. West J Nurs Res 39(6):745-762. https://doi.org/10.1177/0193945916660527

17. Chen S-C, Chou C-C, Chang H-J, Lin M-F (2017) Comparison of group vs self-directed music interventions to reduce chemotherapyrelated distress and cognitive appraisal: an exploratory study. Support Care Cancer 26:1-9. https://doi.org/10.1007/s00520-0173850-1

18. Beaulieu-Boire GMD, Bourque SMD, Chagnon FM, Chouinard LB, Gallo-Payet NP, Lesur OMDP (2013) Music and biological stress dampening in mechanically-ventilated patients at the intensive care unit ward - a prospective interventional randomized crossover trial. J Crit Care 28(4):442-450. https://doi.org/10.1016/ j.jcrc.2013.01.007

19. Nilsson U (2011) Music: a nursing intervention. Eur J Cardiovasc Nurs 10(2):73-74. https://doi.org/10.1016/j.ejcnurse.2010.06.004

20. Vanderlei LCM, Pastre CM, Hoshi RA, de Carvalho TD, de Godoy MF (2009) Basic notions of heart rate variability and its clinical applicability. Rev Bras Cir Cardiovasc 24(2):205-217

21. Karvinen KH, Murray NP, Arastu H, Allison RR (2013) Stress reactivity, health behaviors, and compliance to medical care in breast cancer survivors. Oncol Nurs Forum 40(2):149-156. https://doi.org/10.1188/13.ONF.149-156

22. Kim DH, Kim JA, Choi YS, Kim SH, Lee JY, Kim YE (2010) Heart rate variability and length of survival in hospice cancer patients. J Korean Med Sci 25(8):1140-1145. https://doi.org/10.3346/jkms. 2010.25.8.1140 
23. Thornton LM, Andersen BL, Blakely WP (2010) The pain, depression, and fatigue symptom cluster in advanced breast cancer: covariation with the hypothalamic-pituitary-adrenal axis and the sympathetic nervous system. Health Psychol 29(3):333-337. https:// doi.org/10.1037/a0018836

24. Arab C, Dias DPM, Barbosa RTA, Carvalho TD, Valenti VE, Crocetta TB, Ferreira M, Abreu LC, Ferreira C (2016) Heart rate variability measure in breast cancer patients and survivors: a systematic review. Psychoneuroendocrinology 68:57-68. https://doi. org/10.1016/j.psyneuen.2016.02.018

25. Zhou X, Ma Z, Zhang L, Zhou S, Wang J, Wang B, Fu W (2016) Heart rate variability in the prediction of survival in patients with cancer: a systematic review and meta-analysis. J Psychosom Res 89:20-25. https://doi.org/10.1016/j.jpsychores.2016.08.004

26. PASS 14 Power Analysis and Sample Size Software (2015) NCSS. LLC. Kaysville, Utah, USA. ncss.com/software/pass

27. Robb SL, Burns DS, Carpenter JS (2011) Reporting guidelines for music-based interventions. Music Med 3(4):271-279. https://doi. org/10.1177/1943862111420539

28. Lai H-L, Li Y-M, Lee L-H (2012) Effects of music intervention with nursing presence and recorded music on psychophysiological indices of cancer patient caregivers. J Clin Nurs 21(5-6):745-756. https://doi.org/10.1111/j.1365-2702.2011. 03916.x

29. Stein KD, Jacobsen PB, Blanchard CM, Thors C (2004) Further validation of the multidimensional fatigue symptom inventoryshort form. J Pain Symptom Manag 27(1):14-23. https://doi.org/ 10.1016/j.jpainsymman.2003.06.003

30. Stein KD, Martin SC, Hann DM, Jacobsen PB (1998) A multidimensional measure of fatigue for use with cancer patients. Cancer Pract 6(3):143-152. https://doi.org/10.1046/j.1523-5394.1998. 006003143.x

31. Buysse DJ, Reynolds CF, Monk TH, Berman SR, Kupfer DJ (1989) The Pittsburgh Sleep Quality Index: a new instrument for psychiatric practice and research. Psychiatry Res 28(2):193-213. https:// doi.org/10.1016/0165-1781(89)90047-4

32. Tzeng JI, Fu Y-W, Lin C-C (2012) Validity and reliability of the Taiwanese version of the Pittsburgh Sleep Quality Index in cancer patients. Int J Nurs Stud 49(1):102-108. https://doi.org/10.1016/j. ijnurstu.2011.08.004

33. Zigmond AS, Snaith RP (1983) The hospital anxiety and depression scale. Acta Psychiatr Scand 67(6):361-370

34. Snaith RP (2003) The hospital anxiety and depression scale. Health Qual Life Outcomes 1(1):1-4. https://doi.org/10.1186/1477-7525-1-29

35. Bagri DR, Gupta A, Dube A, Gupta R (2016) Assessment of examination stress and HRV in adolescents. Natl J Integr Res Med 7(4):1-7
36. Hsu H-T, Lin K-C, Wu L-M, Juan C-H, Hou M-F, Hwang S-L, Liu Y, Dodd MJ (2017) Symptom cluster trajectories during chemotherapy in breast cancer outpatients. J Pain Symptom Manag 53(6): 1017-1025. https://doi.org/10.1016/j.jpainsymman.2016.12.354

37. de Jong N, Kester ADM, Schouten HC, Abu-Saad HH, Courtens AM (2006) Course of fatigue between two cycles of adjuvant chemotherapy in breast cancer patients. Cancer Nurs 29(6):467-477. https://doi.org/10.1097/00002820-200611000-00007

38. McClean S, Bunt L, Daykin N (2012) The healing and spiritual properties of music therapy at a cancer care center. J Altern Complement Med 18(4):402-407. https://doi.org/10.1089/acm. 2010.0715

39. Miller AH, Ancoli-Israel S, Bower JE, Capuron L, Irwin MR (2008) Neuroendocrine-immune mechanisms of behavioral comorbidities in patients with cancer. J Clin Oncol 26(6):971-982. https:// doi.org/10.1200/jco.2007.10.7805

40. Liu L, Rissling M, Natarajan L, Fiorentino L, Mills PJ, Dimsdale JE, Sadler GR, Parker BA, Ancoli-Israel S (2012) The longitudinal relationship between fatigue and sleep in breast cancer patients undergoing chemotherapy. Sleep 35(2):237-245. https://doi.org/ 10.5665/sleep. 1630

41. Bro ML, Jespersen KV, Hansen JB, Vuust P, Abildgaard N, Gram J, Johansen C (2018) Kind of blue: a systematic review and metaanalysis of music interventions in cancer treatment. PsychoOncology 27(2):386-400. https://doi.org/10.1002/pon.4470

42. Lafçi D, Öztunç G (2015) The effect of music on the sleep quality of breast cancer patients. Int J Caring Sci 8(3):633-640

43. Crosswell AD, Lockwood KG, Ganz PA, Bower JE (2014) Low heart rate variability and cancer-related fatigue in breast cancer survivors. Psychoneuroendocrinology 45:58-66. https://doi.org/ 10.1016/j.psyneuen.2014.03.011

44. Fagundes CP, Murray DM, Hwang BS, Gouin J-P, Thayer JF, Sollers JJ, Shapiro CL, Malarkey WB, Kiecolt-Glaser JK (2011) Sympathetic and parasympathetic activity in cancer-related fatigue: more evidence for a physiological substrate in cancer survivors. Psychoneuroendocrinology 36(8):1137-1147. https://doi.org/10. 1016/j.psyneuen.2011.02.005

45. Thayer JF, Sternberg E (2006) Beyond heart rate variability. Ann N Y Acad Sci 1088(1):361-372. https://doi.org/10.1196/annals.1366. 014

Publisher's note Springer Nature remains neutral with regard to jurisdictional claims in published maps and institutional affiliations. 